\title{
A!
}

This is an electronic reprint of the original article.

This reprint may differ from the original in pagination and typographic detail.

Mannila, E. T.; Maisi, V. F.; Pekola, J. P.

\section{Self-Calibrating Superconducting Pair-Breaking Detector}

Published in:

Physical Review Letters

DOI:

10.1103/PhysRevLett.127.147001

Published: 01/10/2021

Document Version

Publisher's PDF, also known as Version of record

Please cite the original version:

Mannila, E. T., Maisi, V. F., \& Pekola, J. P. (2021). Self-Calibrating Superconducting Pair-Breaking Detector.

Physical Review Letters, 127(14), [147001]. https://doi.org/10.1103/PhysRevLett.127.147001

This material is protected by copyright and other intellectual property rights, and duplication or sale of all or part of any of the repository collections is not permitted, except that material may be duplicated by you for your research use or educational purposes in electronic or print form. You must obtain permission for any other use. Electronic or print copies may not be offered, whether for sale or otherwise to anyone who is not an authorised user. 


\title{
Self-Calibrating Superconducting Pair-Breaking Detector
}

\author{
E. T. Mannila $\odot,{ }^{1, *}$ V. F. Maisi $\odot{ }^{2}$ and J. P. Pekola $\odot^{1}$ \\ ${ }^{1}$ QTF Centre of Excellence, Department of Applied Physics, Aalto University, FI-00076 Aalto, Finland \\ ${ }^{2}$ Physics Department and NanoLund, Lund University, Box 118, 22100 Lund, Sweden
}

(Received 5 February 2021; revised 23 June 2021; accepted 6 August 2021; published 30 September 2021)

\begin{abstract}
We propose and experimentally demonstrate a self-calibrating detector of Cooper pair depairing in a superconductor based on a mesoscopic superconducting island coupled to normal metal leads. On average, exactly one electron passes through the device per broken Cooper pair, independent of the absorber volume, device, or material parameters. The device operation is explained by a simple analytical model and verified with numerical simulations in quantitative agreement with experiment. In a proof-of-concept experiment, we use such a detector to measure the high-frequency phonons generated by another, electrically decoupled superconducting island, with a measurable signal resulting from less than $10 \mathrm{fW}$ of dissipated power.
\end{abstract}

DOI: 10.1103/PhysRevLett.127.147001

Introduction.-A key prediction of the Bardeen-CooperSchrieffer theory of superconductivity is the existence of an energy gap $\Delta$ for single-particle excitations. The resulting exponentially suppressed density of thermal excitations makes superconductors very sensitive to radiation at frequencies higher than $2 \Delta / h$, with $h$ the Planck constant, which, although detrimental for superconducting circuits used in quantum computing [1-3], enables applications as detectors. Pair-breaking superconducting detectors, such as superconducting tunnel junction [4], kinetic inductance [5,6], and quantum capacitance [7] detectors, have found use in physics and astronomy, enabling single-photon detection at optical [8] and terahertz [7] frequencies. They can also be used as phonon-mediated detectors $[9,10]$, and tunnel junction detectors have been used for phonon spectroscopy [11-14].

In all of these devices, inferring the number of broken Cooper pairs from the measured response requires calibration or modeling. In contrast, in this Letter we present a mesoscopic pair-breaking detector whose response is given simply by a current

$$
I=e \Gamma_{\mathrm{pb}},
$$

where $e$ is the elementary charge and $\Gamma_{\mathrm{pb}}$ is the rate at which Cooper pairs are broken. We describe the device operation with a simple analytical model, which agrees with the predictions of a full numerical model in quantitative agreement with experiment. In our proof-of-concept experiment, we measure the response of the detector to pair-breaking phonons emitted by another superconducting island, while ruling out that the response could be due to non-pair-breaking mechanisms by comparison with a normal-metallic reference source. We extract the fraction of phonons transmitted from emitter to detector and find that dissipated power as low as $10 \mathrm{fW}$ in the emitter is enough to create a measurable signal in the detector. Although the number of Cooper pairs generated per absorbed phonon or photon depends on the frequency [15], because of its well-defined absorption volume and self-calibrating operation we foresee our device as particularly useful for studying propagation of athermal phonons. This is important in detectors [16-18] as well as applications in quantum information based on superconducting circuits, where phonons may cause quasiparticle poisoning over large distances $[19,20]$.

Operating principle.-Our device, a superconducting island with charging energy $E_{C}$ smaller than the superconducting gap $\Delta$, is sketched in Fig. 1(a). We operate at low temperatures $k_{B} T \ll E_{C}, \Delta$ such that the probability of thermally excited quasiparticles is negligible. Incident radiation (pink) breaks Cooper pairs in a mesoscopic superconducting aluminum island (blue) at a rate $\Gamma_{\mathrm{pb}}$. The resulting quasiparticle excitations (light blue circles) relax by tunneling to the normal metal leads through the left or right tunnel junctions with a rate $N_{\mathrm{QP}} \Gamma_{\text {tunn }}$ for $N_{\mathrm{QP}}$ excitations on the island. Since relaxation can happen equally likely through either junction, this process carries no current but sets the time-averaged quasiparticle population to

$$
\left\langle N_{\mathrm{QP}}\right\rangle=\Gamma_{\mathrm{pb}} / \Gamma_{\text {tunn }} .
$$

As quasiparticles can tunnel out as both electrons and holes, the tunneling events occur between the lowestenergy charge states with $N=0,1$ excess electrons on the island, when the gate offset is close to charge degeneracy, $n_{g} \approx 0.5$. In our model, the energy cost $\Delta$ of creating quasiparticles is accounted for by explicitly tracking the number of quasiparticles $N_{\mathrm{QP}}$ on the island [21]. 


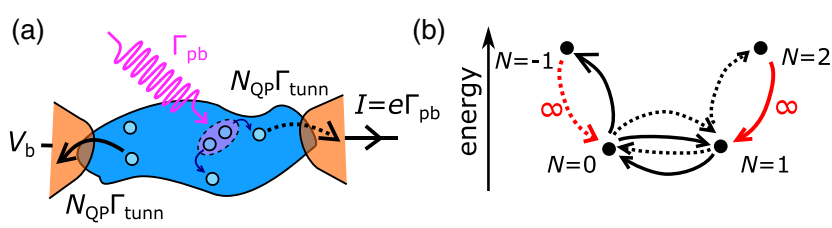

(c)

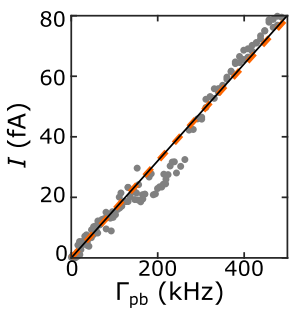

(d)

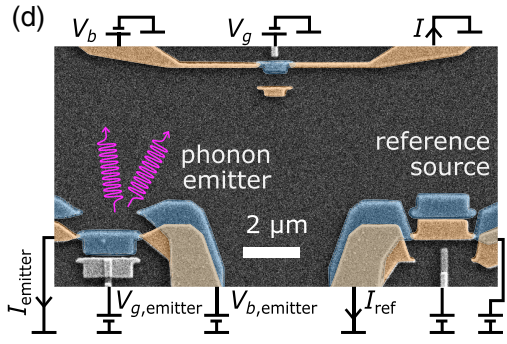

FIG. 1. (a) Sketch of device. Cooper pairs are broken when radiation is absorbed in a superconducting island, and the resulting quasiparticles decay by tunneling to the normal metal leads (black arrows) with a rate $N_{\mathrm{QP}} \Gamma_{\text {tunn }}$ for $N_{\mathrm{QP}}$ excitations on the island. (b) Energies of states with $N=-1,0,1,2$ excess electrons compared to charge neutrality on the island, when the gate offset is close to $n_{g}=0.5$. Black arrows indicate transitions that remove a quasiparticle through the left (solid lines) or right (dashed lines) tunnel junction, while red arrows indicate transitions that add a quasiparticle into the island. The quasiparticle population is set by transitions between charge states $N=0,1$, while net current flows via cycles involving the excited charge states $N=-1,+2$. (c) Numerical verification of operation. The ideal response $I=e \Gamma_{\mathrm{pb}}$ (solid line) is reproduced by our simulations (dashed line) within $1 \%$ up to $\Gamma_{\mathrm{pb}}=500 \mathrm{kHz}$. Symbols show the experimental response, where $\Gamma_{\mathrm{pb}}$ is linearly proportional to the measured $I_{\text {emitter }}$ (d) Proof-of-concept experiment and sketch of measurement setup, with pair-breaking detector (top), superconducting phonon emitter (bottom left), and normal-metallic reference source (bottom right).

In the presence of a small applied bias voltage $V_{b}<\Delta / e$, quasiparticles can also tunnel out to the higher-energy charge states $N=-1$ (2) through the right (left) tunnel junction with the same rate $N_{\mathrm{QP}} \Gamma_{\text {tunn }}$, such that charge is transported in the direction of the bias in both cases. Because of the high energy cost of occupying these states, the island will then return to its previous charge state near instantaneously, when a new quasiparticle tunnels in through the left (right) tunnel junction. This occurs again in the direction of the bias, as indicated by the red arrows in Fig. 1(b), leading to one net electron transported through the device. The cycles $N=0 \rightarrow-1 \rightarrow 0$ and $N=1 \rightarrow$ $2 \rightarrow 1$ do not change the quasiparticle population on the island, yet they determine the current through the device. The total current is set by the rate of the first step of the cycles $N_{\mathrm{QP}} \Gamma_{\text {tunn }}$. Combined with Eq. (2), we obtain the result of Eq. (1): $I=e\left\langle N_{\mathrm{QP}}\right\rangle \Gamma_{\text {tunn }}=e \Gamma_{\mathrm{pb}}$. Here, we have assumed equal tunneling rates in both junctions for simplicity, but the result holds for unequal junctions as well (Supplemental Material [22]). Because the quasiparticle tunneling rates are constant over a range of energy [48], the current due to pair breaking forms diamond-shaped plateaus as a function of $V_{b}$ and $n_{g}$. Although the size and location of these plateaus depends on $E_{C}$, they exist for all $E_{C}<\Delta$ (Supplemental Material [22]) and neither $E_{C}, V_{b}$, nor $n_{g}$ need to be tuned precisely for the self-calibrating operation.

We validate this simple picture by performing numerical simulations based on a rate equation tracking the occupation probabilities of states with $N$ excess electrons and $N_{\mathrm{QP}}$ quasiparticle excitations on the island [21]. The simulations incorporate single-electron and Andreev tunneling at finite temperature of the normal metal leads, as well as quasiparticle recombination through the electron-phonon coupling [21], (Supplemental Material [22]). We find that with our device parameters, the effect of finite temperature and Andreev tunneling is negligible around $n_{g}=0.5$ and $\left|V_{b}\right|<150 \mu \mathrm{V}$. The nonzero electron-phonon recombination rate, scaling as $\Gamma_{\mathrm{R}} N_{\mathrm{QP}}^{2}$ with the prefactor $\Gamma_{\mathrm{R}}$ depending on the device parameters, reduces the quasiparticle population from the value of Eq. (2) and the current response. The condition that recombination be negligible compared to relaxation by tunneling, $\Gamma_{\mathrm{R}}\left\langle N_{\mathrm{QP}}\right\rangle^{2} \ll \Gamma_{\text {tunn }}\left\langle N_{\mathrm{QP}}\right\rangle$, can be expressed as

$$
\Gamma_{\mathrm{pb}} \ll \frac{12 \zeta(5) k_{B}^{5}}{\Sigma \mathcal{V} \Delta^{2} e^{4} R_{T}^{2}}
$$

where $\Sigma$ is the electron-phonon coupling constant, $\mathcal{V}$ is the absorber volume, $\zeta$ is the Riemann zeta function, and $k_{B}$ is the Boltzmann constant (Supplemental Material [22]). For our device parameters, the right-hand side evaluates to $70 \mathrm{MHz}$, corresponding to femtowatts of absorbed power. The simulated current is within $1 \%$ of the ideal value up to $500 \mathrm{kHz}$, the value of $\Gamma_{\mathrm{pb}}$ reached in the experiment, as shown in Fig. 1(c).

Proof-of-concept experiment.-A scanning electron micrograph of our proof-of-concept device is shown in Fig. 1(d). The pair-breaking detector is an aluminum island with $\mathcal{V}=0.9 \times 0.4 \times 0.08 \mu \mathrm{m}^{3}, \quad \Delta=200 \mu \mathrm{eV}$, and $E_{C}=92 \mu \mathrm{eV}$, tunnel coupled to normal metallic copper leads. We fabricate a superconducting phonon emitter and normal metallic reference source on a conducting silicon substrate simultaneously with the detector. The conducting substrate is not necessary for detector operation. Measurements were performed in a plastic dilution refrigerator at a base temperature of $40 \mathrm{mK}$. In measurements with the aluminum in the normal state, we extract device parameters, including the electron-phonon coupling constant $\Sigma_{\mathrm{Al}} \approx 2 \times 10^{8} \mathrm{~W} \mathrm{~K}^{-5} \mathrm{~m}^{-3}$, and verify that heat conduction through the substrate by thermal phonons is negligible as long as the dissipated power is below $1 \mathrm{pW}$ (Supplemental Material [22]).

Figure 2 presents the operation of the self-calibrating detector in the superconducting state. When the current 
experiment

(a) $I_{\text {emitter }}=0$

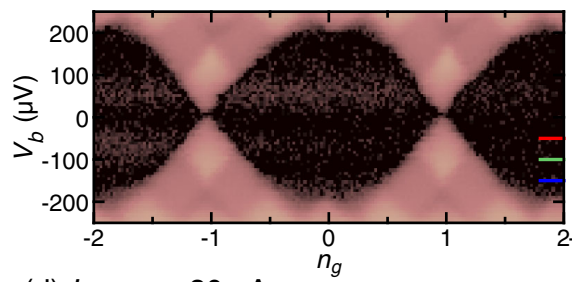

(d) $I_{\text {emitter }}=30 \mathrm{pA}$

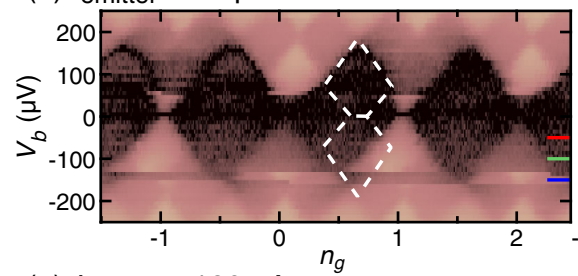

(g) $I_{\text {emitter }}=120 \mathrm{pA}$

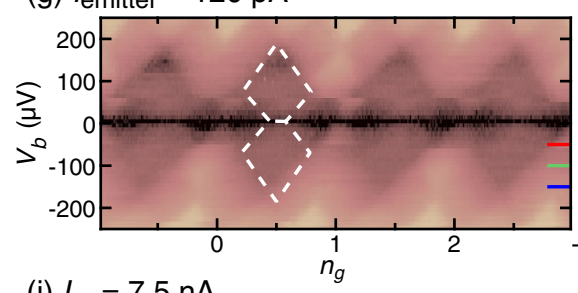

(j) $I_{\text {ref }}=7.5 \mathrm{nA}$

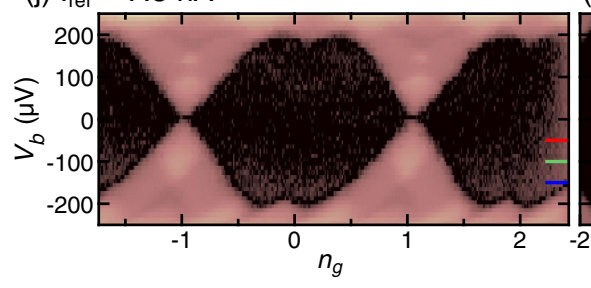

simulation

(b) $\Gamma_{\mathrm{pb}}=50 \mathrm{~Hz}$

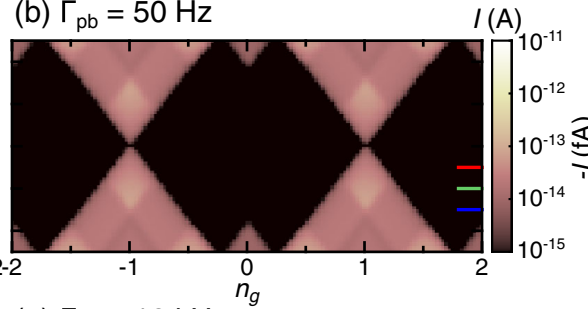

(e) $\Gamma_{\mathrm{pb}}=12 \mathrm{kHz}$ experiment+simulation

(c) $I_{\text {emitter }}=0, \Gamma_{\mathrm{pb}}=50 \mathrm{~Hz}$

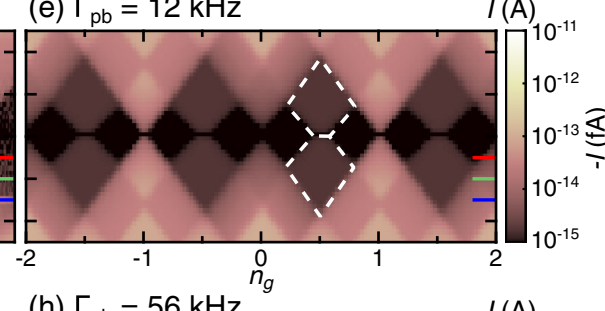

(h) $\Gamma_{\mathrm{pb}}=56 \mathrm{kHz}$

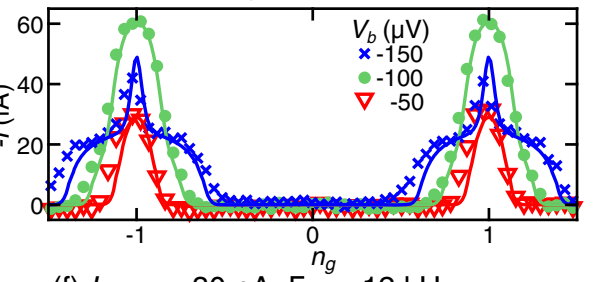

(f) $I_{\text {emitter }}=30 \mathrm{pA}, \Gamma_{\mathrm{pb}}=12 \mathrm{kHz}$

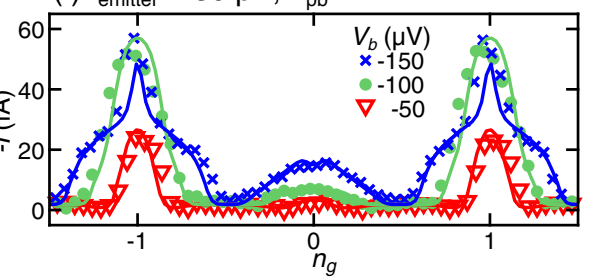

(i) $I_{\text {emitter }}=120 \mathrm{pA}, \Gamma_{\mathrm{pb}}^{g}=56 \mathrm{kHz}$

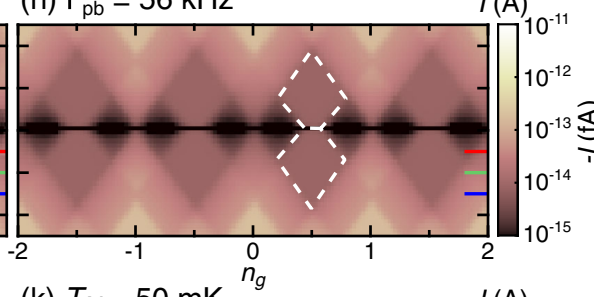

(k) $T_{N}=50 \mathrm{mK}$

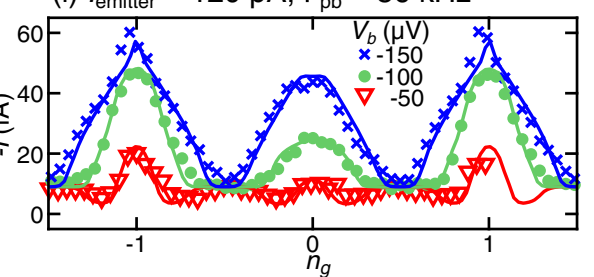

(I) $I_{\mathrm{ref}}=7.5 \mathrm{nA}, T_{N}=50 \mathrm{mK}$
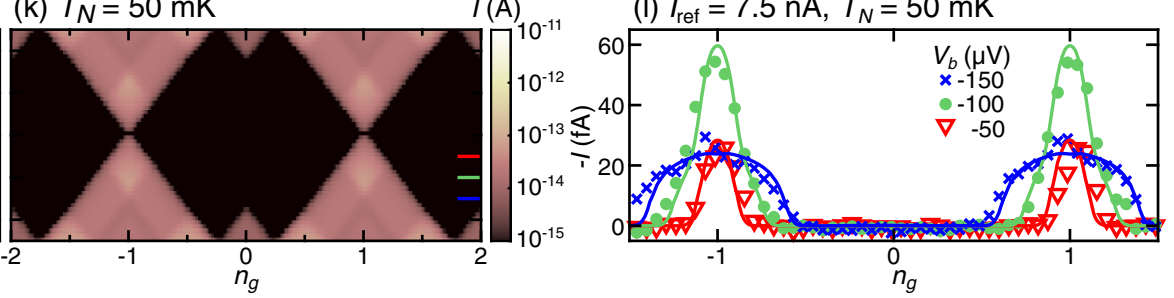

FIG. 2. Measured (left column and symbols in right column) and simulated (middle column and solid lines in right column) subgap current through our pair-breaking detector. On the first row, no current is applied to the emitter or reference source, while the current increases substantially when the current through the phonon emitter is increased to $30 \mathrm{pA}$ (second row) or $120 \mathrm{pA}$ (third row). This is reproduced quantitatively in the simulations by changing only the rate of pair-breaking radiation $\Gamma_{\mathrm{pb}}$. In contrast, increasing the current through the reference emitter up to $7.5 \mathrm{nA}$ (bottom row) creates nearly no change in the detector response, proving that the response is indeed due to pair-breaking phonons. White dashed lines in panels $(\mathrm{d}),(\mathrm{e}),(\mathrm{g}),(\mathrm{h})$ indicate the regions where the self-calibrating response is expected. The rightmost column shows cuts in the data at $V_{b}=-50 \mu \mathrm{V},-100 \mu \mathrm{V}$, and $-150 \mu \mathrm{V}$, also indicated by colored lines in the left and middle columns.

through both the phonon emitter and the reference source is zero, the current at $n_{g}=0.5$ and low $V_{b}$ is zero within the measurement accuracy of roughly $1 \mathrm{fA}$ [Figs. 2(a)-2(c)], as expected. At around $n_{g}=1$, the current is finite due to Andreev reflection [49,50]. When we increase the current through the phonon emitter to $30 \mathrm{pA}$ [Figs. 2(d)-2(f)] or 120 pA [Figs. 2(g)-2(i)], the current level at the plateau around $n_{g}=0.5$ and $\left|V_{b}\right| \approx 100 \mu \mathrm{V}$ increases, as predicted by our simple model. The current increases also around $n_{g}=0$, which is due to Andreev current flowing once the odd charge states are populated due to quasiparticles [51,52].

In contrast, when we increase the current through the reference source up to $7.5 \mathrm{nA}$, while the phonon emitter is kept grounded [Figs. 2(j)-2(l)], the measured current stays zero within the $2 e$-periodic Coulomb diamonds. The main difference between the phonon emitter and the reference source is that the emitter island is superconducting aluminum, which will emit phonons whose energy distribution is peaked above $2 \Delta \approx k_{B} \times 4.6 \mathrm{~K}$ when overheated even slightly [53]. The reference island is normal metallic copper, which will instead emit a broad thermal distribution of phonons, and the superconducting leads of the reference source are connected to their normal metal shadow copies acting as quasiparticle traps. Hence the reference source will emit orders of magnitude fewer phonons with energy larger than $2 \Delta$ than the phonon emitter. This proves that the measured response is indeed due to pair-breaking radiation, unlike in other detector proposals utilizing similar devices [52,54,55].

Our experimental data are quantitatively reproduced by numerical simulations, shown in Figs. 2 and 3. We obtain 


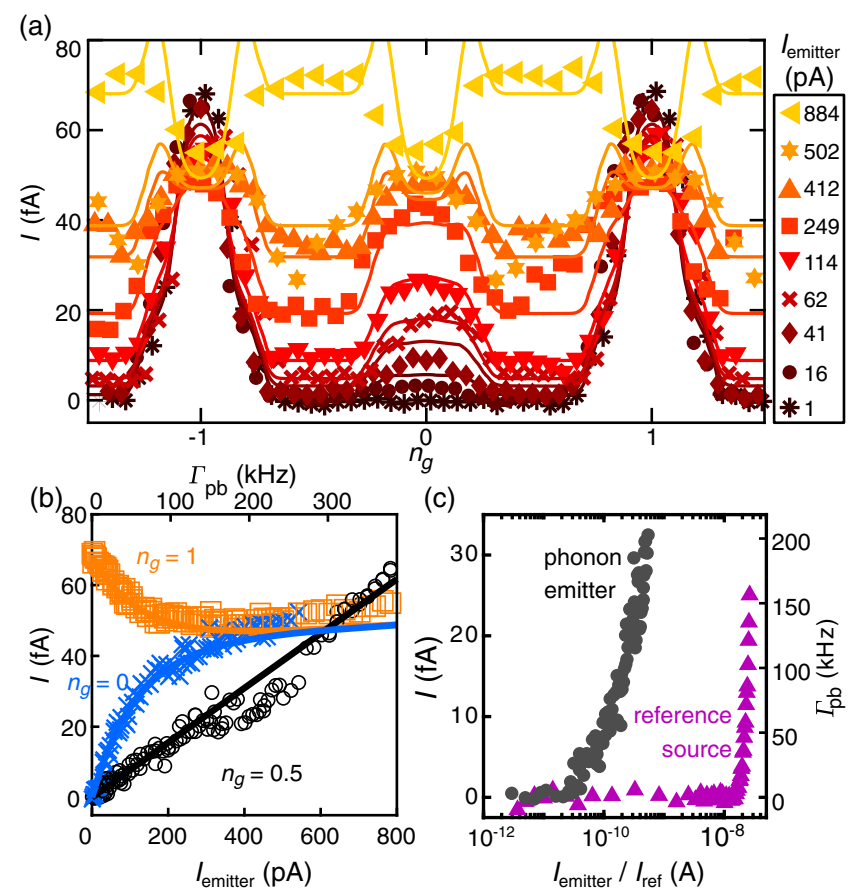

FIG. 3. (a) Detector current vs gate offset $n_{g}$ at $V_{b}=110 \mu \mathrm{V}$ with varying $I_{\text {emitter }}$ in the experiment (symbols) and different Cooper pair-breaking rates $\Gamma_{\mathrm{pb}}=A I_{\mathrm{emitter}} / e$ in the simulations (solid lines). $A=7.8 \times 10^{-5}$ is used for all simulated curves. (b) $I$ extracted at the self-calibrating operating point ( $n_{g}=0.5$, circles) and for comparison at $n_{g}=1$ and $n_{g}=0$, where the current is due to Andreev reflection. Simulated curves (solid lines) for all three values of $n_{g}$ are calculated assuming $\Gamma_{\mathrm{pb}}=A I_{\mathrm{emitter}} / e$ with $A=7.8 \times 10^{-5}$. (c) Measured $I$ at $n_{g}=0.5$ and the corresponding pair-breaking rate in the detector, vs current through either the phonon emitter (black circles) or reference source (magenta triangles).

excellent agreement by changing only $\Gamma_{\mathrm{pb}}$ for differing $I_{\text {emitter }}$. In Fig. 3(a) we show cuts in the data at $V_{b}=$ $110 \mu \mathrm{eV}$ with differing $I_{\text {emitter }}$. At all but the lowest emitter currents, the data agree well with simulations assuming a linear relation $\Gamma_{\mathrm{pb}}=A I_{\mathrm{emitter}} / e$ with $A=7.8 \times 10^{-5}$. Thus our simulation accounts for all the relevant processes, which verifies that the self-calibrating mode can be used in experiments. The points in Figs. 3(b) and 2(c) where the measured current is lower than expected, and the poorer agreement of the data with the simulations at $I_{\text {emitter }}=$ $502 \mathrm{pA}$ in Fig. 3(b), are most likely due to an external disturbance during part of the sweep [22].

Next, we turn to measure the phonon transmission from the phonon emitter to the detector. Modeling of the recombination phonon emission (Supplemental Material [22]) allows us to estimate the emission rate of phonons with energy $\geq 2 \Delta$ as $\Gamma_{2 \Delta}=\eta I_{\text {emitter }} / e$, with the proportionality constant $\eta=0.68$. We find that the measured detector current is also linear in $I_{\text {emitter }}$ at $n_{g}=0.5$, as shown in Fig. 3(b). Hence we extract the proportion of phonons emitted that are absorbed in the detector as
$x=\Gamma_{\mathrm{pb}} / \Gamma_{2 \Delta} \approx 1.1 \times 10^{-4}$. This fraction is difficult to estimate by other means due to the long mean free paths of the phonons at low temperatures, but the order of magnitude is in line with measurements in Refs. $[13,14]$. We also quantify the difference in Cooper pair breaking caused by the phonon emitter and reference source in Fig. 3(c). The reference source causes no detectable signal until currents of almost $20 \mathrm{nA}$ are applied, even when we obtain a measurable signal from as little as $16 \mathrm{pA}$ passing through the superconducting phonon emitter, which corresponds to less than $10 \mathrm{fW}$ of dissipated power.

As a detector of phonons with energy $2 \Delta$, our device has a noise equivalent power of $3 \times 10^{-18} \mathrm{~W} / \sqrt{\mathrm{Hz}}$. In calculating this figure, we use the measured noise level and assume that $25 \%$ of incident phonons are absorbed in the detector (Supplemental Material [22]). This is more than two orders of magnitude better than in the microscale phonon detectors of Ref. [13], while our device also provides the advantage of a self-calibrating operating mode and well-defined absorption volume. The minimum detectable signal in a $1 \mathrm{~s}$ integration time is $\Gamma_{\mathrm{pb}}=1 \mathrm{kHz}$. The maximum output signal of the detector is limited by Eq. (3), yielding a dynamic range that covers several orders of magnitude. The device performance could be enhanced at other operating points once the response has been calibrated in the self-calibrating mode. In our device, the responsivity at low phonon flux increases by roughly a factor of 2 at $n_{g}=0$ [Fig. 3(b)], and could be increased further at higher $V_{b}$.

Conclusion.-In conclusion, we have proposed and experimentally implemented a mesoscopic superconducting detector of high-frequency radiation leading to Cooper pair depairing. We have used the proof-of-concept device to detect the nonequilibrium phonons emitted by another superconducting detector, while using a reference source to rule out mechanisms other than pair breaking. Because of the well-defined microscale absorption volume and selfcalibrating operation, our detector could be particularly useful for studying athermal phonon propagation, relevant both in the context of low-temperature detectors [16-18] and in mitigating phonon-mediated quasiparticle poisoning of superconducting quantum circuits $[19,20,56]$. As a first step in this direction, we extract the fraction of phonons transmitted from emitter to detector over a distance of $8 \mu \mathrm{m}$. As the detector is easily adaptable to different geometries, our device would be straightforward to integrate to study, e.g., the phonon transmission across a typical qubit chip, or fabricate on the sidewalls of a mesa structure for phonon spectroscopy applications [13]. As low a power as $10 \mathrm{fW}$ dissipated in the phonon emitter caused a measurable increase in the Cooper pair creation rate on the absorber island. Hence phonon-mediated poisoning is a plausible explanation of the charge detector backaction of Refs. [57,58], and our results highlight the importance of avoiding dissipation in superconducting quantum devices. 
Finally, we expect that monitoring the individual quasiparticle relaxation events with a fast charge detector [59] would enable detecting every single phonon or photon absorbed.

We acknowledge useful discussions with O. Maillet and J. T. Peltonen. This work was performed as part of the Academy of Finland Centre of Excellence program (Project No. 312057). We acknowledge the provision of facilities and technical support by Aalto University at OtaNanoMicronova Nanofabrication Centre and OtaNano-Low Temperature Laboratory. E. T. M. and J. P. P. acknowledge financial support from Microsoft. V. F. M. acknowledges financial support from the Swedish National Science Foundation, the QuantERA project 2D hybrid materials as a platform for topological quantum computing, and NanoLund.

*elsa.mannila@aalto.fi

[1] J. M. Martinis, M. Ansmann, and J. Aumentado, Energy Decay in Superconducting Josephson-Junction Qubits from Nonequilibrium Quasiparticle Excitations, Phys. Rev. Lett. 103, 097002 (2009).

[2] G. Catelani, J. Koch, L. Frunzio, R. J. Schoelkopf, M. H. Devoret, and L. I. Glazman, Quasiparticle Relaxation of Superconducting Qubits in the Presence of Flux, Phys. Rev. Lett. 106, 077002 (2011).

[3] A. D. Córcoles, J. M. Chow, J. M. Gambetta, C. Rigetti, J. R. Rozen, G. A. Keefe, M. Beth Rothwell, M. B. Ketchen, and M. Steffen, Protecting superconducting qubits from radiation, Appl. Phys. Lett. 99, 181906 (2011).

[4] P. Lerch and A. Zehnder, Quantum Giaever detectors: STJs, in Cryogenic Particle Detection, edited by C. Enss (Springer, New York, 2005), pp. 217-266.

[5] P. K. Day, H. G. LeDuc, B. A. Mazin, A. Vayonakis, and J. Zmuidzinas, A broadband superconducting detector suitable for use in large arrays, Nature (London) 425, 817 (2003).

[6] J. Zmuidzinas, Superconducting microresonators: Physics and applications, Annu. Rev. Condens. Matter Phys. 3, 169 (2012).

[7] P. M. Echternach, B. J. Pepper, T. Reck, and C. M. Bradford, Single photon detection of $1.5 \mathrm{THz}$ radiation with the quantum capacitance detector, Nat. Astron. 2, 90 (2018).

[8] A. Peacock, P. Verhoeve, N. Rando, A. Van Dordrecht, B. G. Taylor, C. Erd, M. A. C. Perryman, R. Venn, J. Howlett, D. J. Goldie, J. Lumley, and M. Wallis, Single optical photon detection with a superconducting tunnel junction, Nature (London) 381, 135 (1996).

[9] L. J. Swenson, A. Cruciani, A. Benoit, M. Roesch, C. S. Yung, A. Bideaud, and A. Monfardini, High-speed phonon imaging using frequency-multiplexed kinetic inductance detectors, Appl. Phys. Lett. 96, 263511 (2010).

[10] D. C. Moore, S. R. Golwala, B. Bumble, B. Cornell, P. K. Day, H. G. LeDuc, and J. Zmuidzinas, Position and energyresolved particle detection using phonon-mediated microwave kinetic inductance detectors, Appl. Phys. Lett. 100, 232601 (2012).
[11] W. E. Bron, Spectroscopy of high-frequency phonons, Rep. Prog. Phys. 43, 301 (1980).

[12] M. N. Wybourne and J. K. Wigmore, Phonon spectroscopy, Rep. Prog. Phys. 51, 923 (1988).

[13] O. O. Otelaja, J. B. Hertzberg, M. Aksit, and R. D. Robinson, Design and operation of a microfabricated phonon spectrometer utilizing superconducting tunnel junctions as phonon transducers, New J. Phys. 15, 043018 (2013).

[14] J. B. Hertzberg, M. Aksit, O. O. Otelaja, D. A. Stewart, and R. D. Robinson, Direct measurements of surface scattering in Si nanosheets using a microscale phonon spectrometer: Implications for Casimir-limit predicted by Ziman theory, Nano Lett. 14, 403 (2014).

[15] T. Guruswamy, D. J. Goldie, and S. Withington, Quasiparticle generation efficiency in superconducting thin films, Supercond. Sci. Technol. 27, 055012 (2014).

[16] F. Henriques, F. Valenti, T. Charpentier, M. Lagoin, C. Gouriou, M. Martínez, L. Cardani, M. Vignati, L. Grünhaupt, D. Gusenkova, J. Ferrero, S. T. Skacel, W. Wernsdorfer, A. V. Ustinov, G. Catelani, O. Sander, and I. M. Pop, Phonon traps reduce the quasiparticle density in superconducting circuits, Appl. Phys. Lett. 115, 212601 (2019).

[17] K. Karatsu, A. Endo, J. Bueno, P. J. de Visser, R. Barends, D. J. Thoen, V. Murugesan, N. Tomita, and J. J. A. Baselmans, Mitigation of cosmic ray effect on microwave kinetic inductance detector arrays, Appl. Phys. Lett. 114, 032601 (2019).

[18] M. Martinez, L. Cardani, N. Casali, A. Cruciani, G. Pettinari, and M. Vignati, Measurements and Simulations of Athermal Phonon Transmission from Silicon Absorbers to Aluminum Sensors, Phys. Rev. Applied 11, 064025 (2019).

[19] U. Patel, I. V. Pechenezhskiy, B. L. T. Plourde, M. G. Vavilov, and R. McDermott, Phonon-mediated quasiparticle poisoning of superconducting microwave resonators, Phys. Rev. B 96, 220501(R) (2017).

[20] C. D. Wilen, S. Abdullah, N. A. Kurinsky, C. Stanford, L. Cardani, G. D'Imperio, C. Tomei, L. Faoro, L. B. Ioffe, C. H. Liu, A. Opremcak, B. G. Christensen, J. L. DuBois, and R. McDermott, Correlated charge noise and relaxation errors in superconducting qubits, Nature (London) 594, 369 (2021)

[21] V. F. Maisi, S. V. Lotkhov, A. Kemppinen, A. Heimes, J. T. Muhonen, and J. P. Pekola, Excitation of Single Quasiparticles in a Small Superconducting Al Island Connected to Normal-Metal Leads by Tunnel Junctions, Phys. Rev. Lett. 111, 147001 (2013).

[22] See Supplemental Material at http://link.aps.org/ supplemental/10.1103/PhysRevLett.127.147001 for additional derivations, details on the numerical simulations, fabrication and measurements, normal-state characterization of the device, and modeling of phonon emission, which includes Refs. [23-47].

[23] J. P. Pekola, V. F. Maisi, S. Kafanov, N. Chekurov, A. Kemppinen, Y. A. Pashkin, O.-P. Saira, M. Möttönen, and J. S. Tsai, Environment-Assisted Tunneling as an Origin of the Dynes Density of States, Phys. Rev. Lett. 105, 026803 (2010). 
[24] M. Taupin, E. Mannila, P. Krogstrup, V. F. Maisi, H. Nguyen, S. M. Albrecht, J. Nygård, C. M. Marcus, and J. P. Pekola, InAs Nanowire with Epitaxial Aluminum as a Single-Electron Transistor with Fixed Tunnel Barriers, Phys. Rev. Applied 6, 054017 (2016).

[25] A. P. Higginbotham, S. M. Albrecht, G. Kiršanskas, W. Chang, F. Kuemmeth, P. Krogstrup, T. S. Jespersen, J. Nygård, K. Flensberg, and C. M. Marcus, Parity lifetime of bound states in a proximitized semiconductor nanowire, Nat. Phys. 11, 1017 (2015).

[26] B. Karimi, D. Nikolić, T. Tuukkanen, J. T. Peltonen, W. Belzig, and J. P. Pekola, Optimized Proximity Thermometer for Ultrasensitive Detection, Phys. Rev. Applied 13, 054001 (2020).

[27] A. B. Zorin, The thermocoax cable as the microwave frequency filter for single electron circuits, Rev. Sci. Instrum. 66, 4296 (1995).

[28] F. Giazotto, T. T. Heikkilä, A. Luukanen, A. M. Savin, and J. P. Pekola, Opportunities for mesoscopics in thermometry and refrigeration: Physics and applications, Rev. Mod. Phys. 78, 217 (2006).

[29] R. L. Kautz, G. Zimmerli, and J. M. Martinis, Self-heating in the Coulomb-blockade electrometer, J. Appl. Phys. 73, 2386 (1993).

[30] J. P. Kauppinen and J. P. Pekola, Electron-phonon heat transport in arrays of $\mathrm{Al}$ islands with submicrometer-sized tunnel junctions, Phys. Rev. B 54, R8353 (1996).

[31] M. Meschke, J. P. Pekola, F. Gay, R. E. Rapp, and H. Godfrin, Electron thermalization in metallic islands probed by Coulomb blockade thermometry, J. Low Temp. Phys. 134, 1119 (2004).

[32] L. B. Wang, O.-P. Saira, D. S. Golubev, and J. P. Pekola, Crossover between Electron-Phonon and BoundaryResistance Limits to Thermal Relaxation in Copper Films, Phys. Rev. Applied 12, 024051 (2019).

[33] V. F. Maisi, O.-P. Saira, Y. A. Pashkin, J. S. Tsai, D. V. Averin, and J. P. Pekola, Real-Time Observation of Discrete Andreev Tunneling Events, Phys. Rev. Lett. 106, 217003 (2011).

[34] J. P. Pekola, K. P. Hirvi, J. P. Kauppinen, and M. A. Paalanen, Thermometry by Arrays of Tunnel Junctions, Phys. Rev. Lett. 73, 2903 (1994).

[35] A. M. Savin, J. P. Pekola, D. V. Averin, and V. K. Semenov, Thermal budget of superconducting digital circuits at subkelvin temperatures, J. Appl. Phys. 99, 084501 (2006).

[36] A. V. Feshchenko, O. P. Saira, J. T. Peltonen, and J. P. Pekola, Thermal conductance of $\mathrm{Nb}$ thin films at sub-kelvin temperatures, Sci. Rep. 7, 41728 (2017).

[37] J.-J. Chang and D. J. Scalapino, Kinetic-equation approach to nonequilibrium superconductivity, Phys. Rev. B 15, 2651 (1977).

[38] A. Rothwarf and B. N. Taylor, Measurement of Recombination Lifetimes in Superconductors, Phys. Rev. Lett. 19, 27 (1967).

[39] D. V. Averin and Y. V. Nazarov, Virtual Electron Diffusion During Quantum Tunneling of the Electric Charge, Phys. Rev. Lett. 65, 2446 (1990).

[40] D. V. Averin and Y. V. Nazarov, Single-Electron Charging of a Superconducting Island, Phys. Rev. Lett. 69, 1993 (1992).
[41] H. S. Knowles, V. F. Maisi, and J. P. Pekola, Probing quasiparticle excitations in a hybrid single electron transistor, Appl. Phys. Lett. 100, 262601 (2012).

[42] M. Marín-Suárez, J. T. Peltonen, and J. P. Pekola, Active quasiparticle suppression in a non-equilibrium superconductor, Nano Lett. 20, 5065 (2020).

[43] S. B. Kaplan, C. C. Chi, D. N. Langenberg, J. J. Chang, S. Jafarey, and D. J. Scalapino, Quasiparticle and phonon lifetimes in superconductors, Phys. Rev. B 14, 4854 (1976).

[44] S. B. Kaplan, Acoustic matching of superconducting films to substrates, J. Low Temp. Phys. 37, 343 (1979).

[45] G. C. O'Neil, P. J. Lowell, J. M. Underwood, and J. N. Ullom, Measurement and modeling of a large-area normal-metal/insulator/superconductor refrigerator with improved cooling, Phys. Rev. B 85, 134504 (2012).

[46] A. Mrzyglod and O. Weis, Mean free path of $\mathrm{THz}$ phonons in aluminum films, J. Low Temp. Phys. 97, 275 (1994).

[47] V. M. Bobetic, Evaluation of high-frequency ultrasonic attenuation in superconductors in the Bardeen-CooperSchrieffer theory of superconductivity, Phys. Rev. 136, A1535 (1964).

[48] O.-P. Saira, A. Kemppinen, V. F. Maisi, and J. P. Pekola, Vanishing quasiparticle density in a hybrid $\mathrm{Al} / \mathrm{Cu} / \mathrm{Al}$ singleelectron transistor, Phys. Rev. B 85, 012504 (2012).

[49] F. W. J. Hekking, L. I. Glazman, K. A. Matveev, and R. I. Shekhter, Coulomb Blockade of Two-Electron Tunneling, Phys. Rev. Lett. 70, 4138 (1993).

[50] T. M. Eiles, J. M. Martinis, and M. H. Devoret, Even-Odd Asymmetry of a Superconductor Revealed by the Coulomb Blockade of Andreev Reflection, Phys. Rev. Lett. 70, 1862 (1993).

[51] J. M. Hergenrother, M. T. Tuominen, and M. Tinkham, Charge Transport by Andreev Reflection through a Mesoscopic Superconducting Island, Phys. Rev. Lett. 72, 1742 (1994).

[52] J. M. Hergenrother, J. G. Lu, M. T. Tuominen, D. C. Ralph, and M. Tinkham, Photon-activated switch behavior in the single-electron transistor with a superconducting island, Phys. Rev. B 51, 9407 (1995).

[53] W. Eisenmenger and A. H. Dayem, Quantum Generation and Detection of Incoherent Phonons in Superconductors, Phys. Rev. Lett. 18, 125 (1967).

[54] O. Naaman and J. Aumentado, Narrow-Band Microwave Radiation from a Biased Single-Cooper-Pair Transistor, Phys. Rev. Lett. 98, 227001 (2007).

[55] B. Jalali-Jafari, S. V. Lotkhov, and A. B. Zorin, Detection of on-chip generated weak microwave radiation using superconducting normal-metal SET, Appl. Sci. 6, 35 (2016).

[56] E. Leonard, M. A. Beck, J. Nelson, B. G. Christensen, T. Thorbeck, C. Howington, A. Opremcak, I. V. Pechenezhskiy, K. Dodge, N. P. Dupuis, M. D. Hutchings, J. Ku, F. Schlenker, J. Suttle, C. Wilen, S. Zhu, M. G. Vavilov, B. L. T. Plourde, and R. McDermott, Digital Coherent Control of a Superconducting Qubit, Phys. Rev. Applied 11, 014009 (2019).

[57] J. Männik and J. E. Lukens, Effect of Measurement on the Periodicity of the Coulomb Staircase of a Superconducting Box, Phys. Rev. Lett. 92, 057004 (2004). 
[58] E. T. Mannila, V. F. Maisi, H. Q. Nguyen, C. M. Marcus, and J. P. Pekola, Detecting parity effect in a superconducting device in the presence of parity switches, Phys. Rev. B 100, 020502(R) (2019).
[59] E. T. Mannila, P. Samuelsson, S. Simbierowicz, J. T. Peltonen, V. Vesterinen, L. Grönberg, J. Hassel, V. F. Maisi, and J. P. Pekola, A superconductor free of quasiparticles for seconds, arXiv:2102.00484. 